\title{
LA FLUCTUACIÓN LABORAL DEL PERSONAL \\ DOCENTE. UNA MIRADA DESDE EL COMPORTAMIENTO HUMANO
}

\author{
MSc. Yaquelin Puebla González \\ Centro de Estudios para el \\ Perfeccionamiento de la Educación \\ Superior \\ Universidad de la Habana \\ yaquelin@cepes.uh.cu
}

\author{
PhD. José Luis Almuiñas \\ Centro de Estudios para el \\ Perfeccionamiento de la Educación \\ Superior \\ Universidad de La Habana \\ almu@cepes.uh.cu
}

PhD. Euclides Catá Guilarte

Departamento de Sociología

Universidad de La Habana

cata@ffh.uh.cu

Recibido: 14 de junio de 2017

Aceptado: 27 de julio de 2017
Palabras claves: comportamiento humano, personal docente, fluctuación laboral.

Keywords: human behavior, teachers, labor fluctuation.

\section{RESUMEN}

La Educación Superior enfrenta el problema de conseguir personal y conservarlo. Para ello, requiere diseñar y aplicar mecanismos que le permita conocer e influir en el comportamiento del docente y desarrollar objetivos institucionales que garanticen la calidad del claustro de manera sostenida. En el presente trabajo se analizan los principales fundamentos teóricos de docente ante el reto de conseguir y conservar dicho personal. En este sentido, se resalta el valor metodológico que tiene este en este análisis la fluctuación laboral a partir de la teoría del comportamiento humano en el personal.

\begin{abstract}
Higher Education faces the problem of getting staff and keeping it. For this, it requires the design and implementation of mechanisms that allow it to know and influence the behavior of the teacher and to develop institutional objectives that guarantee the quality of the faculty in a sustained way. This paper analyzes the main theoretical foundations of labor fluctuation based on the theory of human behavior in the teaching staff in the face of the challenge of obtaining and retaining such personnel. In this sense, the methodological value of this analysis is highlighted.
\end{abstract}




\section{INTRODUCCIÓN}

La sociedad contemporánea ante una realidad inestable, convulsa y compleja, muestra un mundo que tiene como eje fundamental la magnitud y rapidez del cambio, el cual constituye el imperativo posmoderno que lo define. Entre sus rasgos fundamentales se encuentra la crisis sistémica y la creciente incertidumbre social.

Esta crisis sistémica se profundiza en la formación de burbujas financieras, el cambio climático y el aumento de los males sociales. Los cuales según la agenda 2030, muestran las enormes disparidades en cuanto a las oportunidades, la riqueza y el poder, que se concentra en un grupo no representativo con respecto al total de la población mundial. Es sumamente preocupante el desempleo, en particular entre los jóvenes. Los riesgos mundiales para la salud, el aumento de la frecuencia y la intensidad de los desastres naturales, la escala de los conflictos, el extremismo violento, el terrorismo y las consiguientes crisis humanitarias y desplazamientos (2015), que paradójicamente aumentan junto al avance vertiginoso de la ciencia, la tecnología y la innovación.

La sociedad global, a su vez, necesita para su propia reproducción crear mecanismo de transferencia de la ciencia y la tecnología que se constituyan en sus propios mercados, lo cual se revierte en los países en vías de desarrollados a través de becas, donaciones, asegurando por simpatía la dominación económica y política con la cultural, y convierte al área en yacimiento o banco de profesionales del sistema científico internacional.

El sistema científico internacional sufre una polarización de los patrones de hacer ciencia sobre la base de contextos económicos, políticos y culturales dominados por el gran capital. De ahí el éxodo de científicos en búsqueda de los centros de "ciencia mundial", bajo el espejismo de encontrar mejores condiciones intelectuales y económicas para su práctica. Lo anterior pone en peligro la calidad en la enseñanza superior por el éxodo no planificado del personal docente lo que se evidencia en el aumento sostenido de docentes de alto nivel que fluctúan.

Para los países latinoamericanos, esta crisis, tiene su máxima expresión en el aumento de la brecha entre el norte y el sur, situación que se acrecienta en la insuficiente definición de la identidad cultural asumida como proyecto de desarrollo científico por caminos propios, que unido al neocolonialismo cultural, conforman un cuadro desarticulado de la comunidad científica y las tendencias tecnocráticas del desarrollo en América Latina. En este escenario, la Educación Superior tiene un papel primordial en el mejoramiento y la transformación social, donde la atención a los docentes universitarios, desde la concepción del factor humano, es determinante para enfrentar las consecuencias y dificultades crecientes derivadas de esta situación.

Según Fernández (2012) los problemas actuales que enfrentan las IES latinoamericanas están relacionados también con esta situación, por ejemplo: la privatización de este nivel educacional, caracterizada por la diversificación institucional y la heterogeneidad en los niveles de calidad; la proliferación de instituciones denominadas 
"universidades", pero que desde el punto de vista de la calidad en su oferta no lo son; la supervivencia de modelos académicos y de gestión tradicionales; baja inversión en investigación y desarrollo, con pobre participación del sector privado en este gasto y limitada inversión en la formación de posgrado, particularmente, el doctorado, lo que tiene una implicación en la calidad de la docencia y la investigación; así como las deficiencias en los sistemas de selección, desarrollo, promoción y régimen salarial del personal de dichas instituciones (2012).

Esto último trae como consecuencia el empobrecimiento de la profesión académica en la región en comparación con el desarrollo que ha mostrado en otras partes del mundo.

\section{DESARROLLO}

Ante esta realidad, los problemas antes mencionados no podrán ser solventados si no se brinda una atención integral a dicho personal que quede refrendada como parte de la política institucional, donde la labor del docente adquiera el valor que realmente tiene en la formación de las nuevas generaciones.

Al respecto, la Conferencia Regional sobre Educación Superior (CRES, 2008) pauta que el docente universitario, se erige en actor fundamental del sistema educativo, cuya formación, capacitación permanente, en adecuadas condiciones laborales y regímenes de trabajo, salario y carrera profesional, permiten hacer efectiva la calidad en la enseñanza y la investigación. Se convierte así en una figura central a través del cual se gana o se pierde el prestigio académico.

En ello radica, la importancia que la IES realice ingentes esfuerzos por brindar un mejor tratamiento a este personal, que se traduzca en mejorar las condiciones de trabajo y vida, brindar posibilidades para la formación continua con énfasis en la doctoral y posdoctoral, lo cual facilite una mayor participación en los intercambios académicos que enriquezca su arsenal teórico, metodológico y práctico.

Aun cuando estas actividades comienzan a formar parte de las agendas universitarias, todavía resultan insuficientes. La IES se preocupa por la pérdida de personal docente, sobre todo, del que está mejor formado. Un aspecto central a tener en cuenta en su desarrollo y proyecciones de trabajo es lograr, en lo posible, la conciliación de los objetivos de trabajo con los personales, de manera tal, que puedan atenderse las necesidades, demandas y motivaciones de los docentes universitarios, ya que sobre ellos recae el mayor peso del trabajo y depende el éxito o fracaso de dicha institución.

El objetivo que se propone es analizar los principales fundamentos teóricos de la fluctuación laboral a partir de la teoría del comportamiento humano del personal docente ante el reto de conseguir y conservar dicho personal. En el trabajo se resalta la vigencia e importancia que tiene este tema para la IES. 


\section{El valor metodológico del análisis del comportamiento del personal docente}

Una problemática que pone en peligro la calidad de la enseñanza superior en la actualidad es el flujo no planificado de personal docente, lo que se evidencia en el aumento sostenido de la fluctuación laboral de los mismos, que en su mayoría tienen alto nivel de calificación. En este proceso se involucran un conjunto de factores que van desde la visibilidad por parte de la institución de conseguir y conservar sus profesionales hasta la concientización de estos actores acerca del papel que desempeñan. El comportamiento de los docentes en la institución se convierte en una herramienta para el análisis de la fluctuación laboral y el mejoramiento de la gestión de dicho personal que tribute a su conservación.

El comportamiento del docente universitario es muy variable y depende de cómo se relacionan numerosos factores sociales, genéticos, neuro-hormonales, psicológicos y de la biografía del individuo, que tienen su explicación en la teoría del comportamiento organizacional.

Es aquí cuando la idea de cadena causal y la interacción entre elementos de distinto orden cobran especial importancia, en particular, para comprender e influir en las decisiones de continuidad o fluctuación.

En las investigaciones realizadas sobre este tema numerosos autores comparten el criterio de entender el comportamiento como "el resultado de cuatro componentes: el individuo, el grupo, la organización y lo social". (Romero, 2008). El estudio de cada uno aporta información sobre los elementos esenciales a tener en cuenta para comprender la actuación del docente en la IES.

La gestión del personal docente se inicia en el nivel individual. Su análisis parte del supuesto que el mismo, una vez que decide incorporarse a la IES, pierde su individualidad para convertirse en estructura de la institución. (Romero, 2008). Esto se explica mediante la acción de teorizar al docente para un puesto, un cargo, un proceso o un área, a través de la selección. Una vez que se toma la decisión de quién ocupará el puesto, las características individuales desaparecen por la acción social e institucional. El problema entonces radica en lograr que este profesional se comporte de acuerdo a lo esperado. Si esa conducta no se logra, la IES generalmente prescinde de él.

Las contribuciones de los representantes más destacados en este nivel culminan en teorías que evolucionan para explicar el grado de implicación de los docentes en las IES, realizando aportes teóricos desde la Psicología Social y la Sociología. Las variables en este nivel toman en consideración al aspecto intelectual (la percepción), el emocional (la motivación) y las características de la personalidad.

El análisis de estas variables parte de considerar al docente como ente bio-psico-social. En esencia se defiende la idea que este profesional, como individuo, tiene en sí todas las potencialidades del universo, es decir, él es universo y su atención debe estar encaminada a tener en cuenta esta condición holística que le hace merecedor 
de un tratamiento integral. Para ello, el análisis de esta condición se inicia con la percepción, primera variable que nos va a permitir la identificación y comprensión de los motivos de su fluctuación.

La percepción es un proceso cognitivo mediante el cual los docentes reúnen información del medio, a fin de darle un significado a su ambiente. Está relacionada con la imagen, las elecciones y decisiones. Uno de los representantes de esta corriente es Herbert A. Simón con la perspectiva estructural de toma de decisiones organizacional. Importante en esta teoría es el concepto de racionalidad limitada, que se centra en la búsqueda y selección de alternativas satisfactorias, no óptimas, apoyadas por la institución al crear un entorno psicológico o cognitivo que asegura la correcta toma de decisiones y la "integración de distintos puntos de vista, relacionados con los aspectos instrumentales del comportamiento humano, la motivación, la actitud y la razón". (López, 2013).

En esta misma variable se considera importante el balance entre las compensaciones y las contribuciones de la institución al docente y viceversa. Este depende tanto de la deseabilidad percibida de dejar el puesto, lo que se relaciona con la satisfacción en el trabajo, como de la factibilidad percibida de abandono, que se relaciona con el número de alternativas percibidas fuera de la IES. (López, 2013). Este análisis puede contribuir a realizar el balance de costos beneficios en relación a la decisión de conservación de este personal.

Por su parte las emociones, se relacionan a la parte afectiva y tienen su explicación en las diversas teorías de la motivación. En la actualidad existe una tendencia a reunir una variedad conceptual y teórica sobre la motivación. Lo que en criterio de Robbins y Judge (2009) es pertinente para lograr su interpretación en el trabajo bajo un prisma integrado. De manera general se trata de reunir en uno, los enfoques de la motivación extrínseca y en otro los enfoques de la motivación intrínseca. Se enfatiza en algunos aspectos en dependencia del modelo a seguir.

La motivación es considerada un proceso interno de impulso, relacionado con algún evento interno o externo, que se incorpora en la manera como se asume el comportamiento frente al trabajo, los demás profesionales y la institución. Se clasifica en personales, laborales y sociales, que hacen referencia a los factores que determinan las conductas de los docentes en el momento de la fluctuación y se explican a partir del comportamiento causado y orientado hacia objetivos.

En el caso de las características de los docentes se tienen en cuenta la edad, el sexo, la categoría y las competencias profesionales. El análisis de la edad se realiza tomando dos grupos de referencia: los más jóvenes con mayor probabilidad de fluctuar y los de mayor experiencia, más estables, comprometidos y cerca de la jubilación. Ambos grupos necesitan de una estrategia específica institucional que favorezca su conservación. En esta variable un aspecto importante a considerar es la brecha generacional con la carencia de reemplazo. 
En cuanto al sexo, tiene menores niveles de generalidad, aunque existen casos que las instituciones prefieren contratar a los hombres, porque tienen roles diferentes en cuanto al cuidado familiar y son más estables en el trabajo. Con respecto a la categoría se prefiere al docente mejor formado, de manera tal que la institución tenga que invertir lo menos posible y en cuanto a la competencia profesional se recomienda que sea constantemente motivada y entrenada.

En este nivel además de la consideración del docente como un ente bio-psico-social, está el hecho que se encuentra inmerso en las relaciones que se dan en la institución. En este sentido, él es relación y a partir de lo que establece en sus interacciones con los demás se forma en reestructuración la institución y en un nivel mayor; esa relación es expresión de la sociedad si existe la suficiente complejidad y diferenciación.

Como expresión de esa vinculación, el análisis a nivel grupal parte de considerar todas las variables anteriormente mencionadas. En la medida que se reproducen un conjunto de actitudes y conductas, reflejo de la cultura de procedencia, se crean los grupos informales, donde la interacción se constituye en el elemento central que se da en la medida que "las personas actúan según el modo en el que perciben que ellas mismas interaccionan con el entorno". (Romero, 2010).

El análisis del comportamiento a nivel grupal permite articular dos extremos teóricos que explican la conducta de los docentes. En uno, se encuentran las perspectivas de la psicología individual que proponen la explicación del comportamiento de los mismos como un problema individual, y en el otro, las posiciones sociales que incluyen la antropología, la sociología, y en general, las ciencias sociales, a favor de la conducta de dicho personal como un problema de origen y de orden social. (Romero, 2010).

En criterio de los autores, el trabajo realizado en grupo refleja, en gran medida, la percepción que sobre la institución tiene el personal docente, así como la motivación a continuar en su puesto, ya que la actividad concreta que realiza está más o menos apoyada por el grupo o el equipo de trabajo, y en esa relación se miden los objetivos alcanzados, el nivel de satisfacción con la actividad realizada y el prestigio profesional que se tiene o se quiere.

El trabajo realizado en el grupo depende sobre todo de los intereses institucionales y esto tiene una expresión en el nivel de análisis organizacional. En este nivel se hace énfasis en los procesos contextuales y relacionales. La IES marca pautas de comportamiento relacionadas con la profesionalidad, el prestigio, sentar precedentes y darse a conocer por sus conocimientos, etc., por lo cual resulta importante el análisis del clima y el entorno social.

El clima o ambiente de trabajo en las instituciones constituye uno de los factores determinantes y facilitadores de los procesos organizativos y de gestión, además de los de innovación y cambio. En la mayoría de las definiciones lo relacionan con el ambiente interno, lo que tiene un impacto en la conducta de los docentes y en el comportamiento organizacional. Es decir, la forma en que este personal percibe el 
clima de la organización resulta clave para comprender su comportamiento en ese contexto.

En el análisis del clima es central el concepto de estructura que según Weber se clasifica como burocrática, a lo que Mintzberg le agrega profesional. La burocracia profesional "se caracteriza por presentar una estructura altamente democratizada tanto a nivel horizontal como vertical y se basa en el poder del experto, lo que significa que los profesionales pueden controlar su propio trabajo y, de algún modo, llegan a tener control de algunas decisiones administrativas". (Marsollier y Aparicio, 2014). Esto es especialmente evidente cuando pensamos en la labor profesional del docente universitario, ya que, dentro de ciertos parámetros, trabaja con relativa independencia de sus colegas, pero en estrecho contacto con los estudiantes.

La estructura está estrechamente ligada a la cultura de la institución educativa. Al decir de Oltra (2011), el conjunto de roles y estatus está conformado por normas y patrones culturales, que van desde ideas generales sobre el bien o el mal hasta reglas de comportamiento específicas que distinguen el modo de ser y hacer de los docentes universitarios. La cultura es un elemento fundamental en el análisis del comportamiento. Para su estudio se identifican las creencias, las normas y los valores predominantes que distinguen la conducta de los docentes en una IES y nos permite indagar en los elementos contextuales de origen ya sea en el orden individual o grupal, lo que nos puede facilitar establecer la cadena de sucesos que inician la fluctuación laboral.

Otro concepto importante para la comprensión del comportamiento del docente en una IES es introducido también por Weber referido al tiempo y el espacio, que de acuerdo con Giddens (2000) se une al control de la información, donde la escritura tiene importancia capital. Esto expresa como una institución precisa de re- glas escritas para funcionar y de archivos en los que se almacene su memoria. La actividad del docente no sería posible sin un número constante de horas de trabajo. La coordinación de dicha actividad de forma coherente en función del tiempo y el espacio, hace en palabras de Foucault, que se distribuyan a los cuerpos eficazmente en la institución. Los horarios son un requisito de la disciplina, porque organizan las actividades formativas e investigativas.

El análisis hasta aquí realizado es incompleto. A los planteamientos anteriores, se agrega el nivel social en el cual se encuentran las IES. La reflexión, la investigación y la construcción de teoría, tuvieron que reestructurar completamente su formulación, ya que se debía incorporar el entorno social y definir cómo este generaría y determinaría la conducta de dicho personal.

El entorno es un concepto clave para comprender la conducta de los docentes universitarios, ya que impacta de manera constante su desarrollo y sus proyecciones a futuro. Uno de los elementos a considerar está relacionado con el desarrollo tecnológico. En este sentido, la IES está cambiando en la medida que su actividad formativa se realiza de manera virtual, lo cual tiene una evolución creciente. Otro aspecto está 
relacionado con las políticas educativas y públicas, así como los cambios y demandas que acontecen en el mundo laboral, donde el docente está obligado a incrementar sus competencias para suplir la exigencia de los educandos.

Todos estos elementos están mediados por el cambio, su rapidez e inmediatez y la dificultad para medirlo, donde resultan clave los factores que impactan el comportamiento y el peso que tiene cada uno de ellos. En palabras de Oltra (2011) desentrañar las claves del cambio en las sociedades es una tarea complicada, por las dificultades en compaginar explicaciones en sus distintos niveles, por la cantidad de fenómenos implicados en el mismo y la dificultad de desentrañar el efecto causal. En dependencia del lugar o factor de la cadena donde nos detengamos a realizar el análisis del comportamiento del docente, la explicación a este fenómeno puede ser diferente.

Lo visto hasta aquí nos acerca al debate actual sobre el origen de la conducta de los docentes. En el centro de la cuestión se encuentran la percepción, la motivación, la interacción, los genes, la estructura, la cultura, el entorno y el cambio como factor determinante de este proceso. Todo ello esta mediado por dos posiciones fundamentales, aquella que se realiza de manera consciente, donde interviene el lado racional y objetivo del docente y la que implica la acción inconsciente, donde intervienen elementos desconocidos desde el punto de vista racional. Ambas posiciones a su vez, están mediadas por las necesidades e intereses de los docentes de ser aceptados socialmente de donde se deriva la conformidad con el cumplimiento de las normas y la búsqueda de una posición o estatus donde afianzarse.

\section{CONCLUSIONES}

De manera general se puede concluir que lo expuesto anteriormente supone una cadena de sucesos e información que inciden en la conducta del personal docente. En este entramado de relaciones convergen diversas teorías que transitan del hombre racional o económico al hombre social y organizacional.

Los conceptos de hombre y relación adquieren una relevancia especial. El docente universitario actúa acorde a dos principios fundamentalmente. Uno, dirigido a obtener el máximo de beneficios posibles para sí mismo, lo cual responde al principio de acción racional y el otro, encaminado al seguimiento de normas sociales, aquellas que tienen como finalidad maximizar el bienestar. Ambos modelos de actuación son recurrentes en cualquiera de los análisis dirigidos a comprender su comportamiento a nivel individual, grupal, organizacional y social.

El problema de conseguir y conservar al personal docente desde la óptica de un dilema comenzó a partir del análisis de la fluctuación laboral. Como se ha mostrado requiere rebasar la mirada de lo individual, ya que cualesquiera que sean los motivos del personal docente para fluctuar, esa decisión refleja su posición en el contexto social, el cual está compuesto de una colección aleatoria de acontecimientos y acciones estructurados de diversas maneras, donde la forma de comportarse y relacionarse presenta regularidades y es a la vez reflejo del ambiente social de procedencia. 


\section{REFERENCIAS BIBLIOGRÁFICAS}

Fernández, N. (2012). La profesión académica en América Latina. Situación y perspectivas en: N., Fernández \& M. Mónica (Comp.), El futuro de la profesión académica: desafíos para los países emergentes, (pp. 61-78), (Universidad Nacional de Tres de Febrero ed.), Buenos Aires/ Argentina.

Giddens, A. (2000). Sociología. In T. e. revisada (Series Ed.) A. E. S.A. (Ed.).

López, M. T. (2013). La cultura organizativa como herramienta de gestión interna y de adaptación al entorno. Un estudio de casos múltiples en empresas murcianas. Universidad de Murcia.

Marsollier, R. G. \& Aparicio, M. (2014). Universidad y factores de riesgo laboral. Una aproximación al desgaste profesional en empleados universitarios. Re- vista de Psicología - Universidad Viña del Mar, Vol. 3, № 6, 24-33.

Oltra, C. (2011). La sociedad al desnudo. Una nueva invitación a la sociología. Editorial CírculoRojo. Extraído el 23 el enero, 2017 de http://studylib.es/doc/8599272/la-sociedad-al-desnudo.-una-nueva-

ONU. (2015). Transformar nuestro mundo: la Agenda 2030 para el Desarrollo Sostenible. Cumbre de Naciones Unidas (p. 6). Nueva York: Naciones Unidas.

Robbins, S. P. \& Judge, T. A. (2009). Comportamiento organizacional (P. Educación Ed. Decimotercera edición ed.) México, p.29.

Romero, D. (2008). La dimensión individual en el comportamiento organizacional. Revista Iberoamericana de Psicología: Ciencia y Tecnología, 1, 51-60.

Romero, D. (2010). La dimensión grupal en el comportamiento organizacional. Revista Iberoamericana de Psicología: ciencia y Tecnología 3(1), 27-38.

UNESCO (2008). Declaración de la Conferencia Regional de Educación Superior en América Latina y el Caribe, p, 6. 